\title{
Case based evidence of compensatory model for Attention deficit hyperactive disorder and online gaming: implications for school hyperactivity health
}

\author{
Manoj Kumar Sharma $^{1 *}$, Nitin Anand ${ }^{2}$, Pranjali Chakraborty Thakur ${ }^{1}$, Ishita Modal ${ }^{1}$, Priya Singh ${ }^{1}$, Ajith SJ ${ }^{1}$, Suma N$^{1}$, Nisha John ${ }^{1}$, Ankita \\ Biswas $^{1}$, Archana R ${ }^{1}$, Akash Vishwakarma ${ }^{1}$, Keshava D Murthy ${ }^{3}$ and Hemant Mondal Gupta ${ }^{4}$ \\ ${ }^{1}$ Clinical Psychology, SHUT clinic (Service for Healthy use of Technology), National Institute of Mental health \& Neurosciences, Bengaluru, Karnataka, India \\ ${ }^{2}$ Clinical Psychology, National Institute of Mental health \& Neurosciences, Bengaluru, Karnataka, India \\ ${ }^{3}$ Department of Psychiatric Social work, NIMHANS, Bangalore, Karnataka, India \\ ${ }^{4}$ CCRAS, NIMHANS, Karnataka, India
}

\begin{abstract}
Attention deficit hyperactivity disorder (ADHD) is the most common psychiatric disorder among adolescents with Internet addiction. This case approached the tertiary speciality centre for management of issues related to gaming. The clinical interview technique was used to elicit the psychopathology and the current usage of technology. The case reflects the lack of screening or management of above-mentioned issues in the school setting. The lack of attention to these issues leads to the use of online modality to feel good among users with ADHD.
\end{abstract}

\section{Introduction}

Internet Use is the most common and popular medium for recreational use among today's' generation. But it does manifest as problematic to addictive use among a certain group of users. Studies from different parts of the world suggest that the overall prevalence of Internet addiction in adolescents ranges between $2 \%$ and $18 \%$.[1] The prevalence of attention deficit hyperactivity disorder (ADHD) in one of the Southern states of India was 5.76\% with 3.8\% among girls and $1.9 \%$ among boys between the age group of 6 to 11 years. Many researchers and clinicians noted that a variety of mental disorders occur with Internet Addiction [2]. Attention deficit hyperactivity disorder(ADHD) is the most common psychiatric disorder among adolescents with Internet addiction who are referred for psychiatric treatment [3]. Attention deficit was the most associated symptom of Internet addiction, followed by impulsivity.[4] The other associations found in ADHD adolescents were high fun-seeking and behavioural inhibition [5] The presented case approached the tertiary speciality centre for management of issues related to gaming.

\section{Case}

Mr R a 16 years old boy presented with a history of inattention, increased activity and excessive time spent on gaming over the last two years. There was a family history of attention deficit disorder in the father Father functioning reported to be grossly inadequate. He used to attend regular school. Due to inattention problems, he had academic difficulty. in addition to school complaints of distracting school mates and not completing school work. This led to a loss of interest in academics and frequent absenteeism from school. He did not have any other leisure activity nor did he have offline friends and spent his free time at home only. The mental health consultation was initiated and he was diagnosed with attention deficit hyperactive disorder.
Behaviour therapy was initiated for management of ADHD which led to improvements in school attendance but the issue of not completing assignments and distracting others persisted. He was introduced to gaming two years ago by his peers. He started enjoying playing Online Multiple Player battle arena games. He started spent significant amount of time in chatting with online friends. He was lauded for his gaming techniques and scores and getting a good rank within short period. Because of this he even contemplated becoming a gamer and had even won few monetary rewards from gaming tournaments. His parents observed improvement in his behaviors whenever he was allowed to play. He gradually expressed resistance to attending school and eventually stopped going to school. The reason that why he was into gaming was the lack of recognition in school, not having friends and feeling guilty over not being able to sustain attention. All these factors also contributed to his solitary lifestyle and boredom. while online gaming, made him feel comfortable due to the stimulating nature of the games and activity involved, not feeling pressurized to perform and getting awards which he did in the offline world. He was in the precontemplation stage for seeking treatment. He did not want to quit nor change his gaming habit. The parent also reported no other behavioural issues besides spending an excessive amount of time on gaming. Clinical interview revealed presence of of craving, loss of control, maladaptive coping modality and behavioural consequences. He did not maintain follow up with clinic and attended 4 sessions. The

${ }^{\star}$ Correspondence to: Manoj Kumar Sharma, Dept of Clinical Psychology, SHUT clinic (Service for Healthy Use of Technology), NIMHANS, Bengaluru, India; E-mail: shutclinic@gmail.com

Key words: $A D H D$, technology, coping

Received: March 15, 2020; Accepted: March 23, 2020; Published: March 30, 2020 
psychological work initiated with parent to give them understanding of gaming addiction, role of ADHD in excessive use of gaming, building up leisure time activities relaxation exercise and problems solving approach was demonstrated to parent.

\section{Discussion and conclusions}

The case reflect the lack of screening or management of these issues in school setting. The lack of attention to these issues leads use of online modality to feel good. It was gaming in this case. Review of literature also suggest use of online activities as a form of coping methods[6,7]. The compensatory internet use theory found that the relationship between stress and excessive online gaming was mediated by escapism, a motivation for the play that is often linked to problematic outcomes. [8] It can be understood in terms of the biopsychosocial mechanism for the coexistence between ADHD and Internet addiction. Firstly, individuals with ADHD are usually easily bored and have an aversion for the delayed reward. The internet provides rapid responses and immediate rewards to reduce the feeling of boredom. Secondly, striatal dopamine is released during video gaming, which may help the players to stay focused on gaming and have better performances. Thirdly, adolescents with ADHD have abnormal brain activities associated with impaired inhibition, which may cause individuals with ADHD to become vulnerable to internet addiction. Fourthly, motivation deficits and less arousal to punishment, are typical features of ADHD. [9][10] Internet activities such as online gaming usually provide immediate response and reward and thus contribute to high vulnerability to internet addiction among people with ADHD. The model proposed that "being easily bored" and "having an aversion for delayed rewards" are the two main ADHD symptoms and internet use provides multiple windows with a variety of activities at the same time and an immediate reward may decrease the boredom feeling and reward quickly. This feature makes people with ADHD addicted to the internet more easily. It is these reasons viz screening and motives to access online gaming needs to be addressed as a preventive strategy in school settings.

\section{Acknowledgement}

Non funded clinic work Dr Manoj Kumar Sharma.

\section{Compliance with ethical standard}

There was no conflict of interest in relation to present work as well as informed consent of the human subjects had been taken prior to inclusion in the study.

\section{Disclosure of potential conflict of interest}

Not applicable.

\section{Conflict of interest}

Authors of the paper did not have any conflict of interest.

\section{Statement of human right}

The studies have been approved by the Institutional and/or national research ethics committee.

\section{Research involving human participants and/or animals}

All procedures performed in studies involving human participants were in accordance with the ethical standards of the institutional and/ or national research committee and with the 1964 Helsinki declaration and its later amendments or comparable ethical standards.

\section{Reference}

1. Petersen KU, Weymann N, Schelb Y, Thiel R, Thomasius R (2009) Pathological Internet use- epidemiology, diagnostics, cooccurring disorders and treatment. Fortschr Neurol Psychiatr 77: 263-271. [Crossref]

2. Cash H, Rae CD, Steel AH, Winkler A (2012) Internet Addiction: A Brief Summary of Research and Practice. Current Psychiatry Reviews 8: 292-298. [Crossref]

3. Bozkurt H, Coskun, M, Ayaydin, H, Adak I, Zoroglu (2013) Prevalence and patterns of psychiatric disorders in referred adolescents with Internet addiction. Psychiatry Clin Neurosci 67: 352-359. [Crossref]

4. Yen JY, Yen CF, Chen CS, Tang TC, Ko CH (2009) The Association between Adult ADHD Symptoms and Internet Addiction among College Students: The Gender Difference. Cyberpsychol Behav 12: 187-191. [Crossref]

5. Chou WJ, Liu TL, Yang P, Yen CF, Hu HF (2015) Multi-dimensional correlates of Internet addiction symptoms in adolescents with attention-deficit/hyperactivity disorder. Psychiatry Res 225: 122-128. [Crossref]

6. Bessière k, Kiesler S, Kraut S, Boneva B (2008) Effects of internet use and social resources on changes in depression Information, Communication and Society 11: $47-70$.

7. Caplan S, High A (2011) Online social interaction, psychosocial well-being, and problematic internet use in K. Young, C. Abreu (Eds.), Internet addiction: A handbook and guide to evaluation and treatment, John Wiley \& Sons Inc, Hoboken, NJ, US, 35-53.

8. Kardefelt-Winther D (2014) Problematizing excessive online gaming and its psychological predictors Computers in Human Behavior 31: 118-122

9. Ko CH, Yen JY, Yen CF, Chen CS, Chen CC (2012) The association between Internet addiction and psychiatric disorder: a review of the literature. Eur Psychiatry 27: 1-8. [Crossref]

10. Berger A, Kofman O, Livneh U, Henik A (2007) Multidisciplinary perspectives on attention and the development of self-regulation. Prog Neurobio 82: 256-286. [Crossref]

Copyright: (C2020 Sharma MK. This is an open-access article distributed under the terms of the Creative Commons Attribution License, which permits unrestricted use, distribution, and reproduction in any medium, provided the original author and source are credited. 